\title{
Glucose- and glycaemic factor-lowering effects of probiotics on diabetes: a meta-analysis of randomised placebo-controlled trials
}

\author{
Jing Sun $^{1 *}$ and Nicholas J. Buys ${ }^{2}$ \\ ${ }^{1}$ School of Medicine, Menzies Health Institute Queensland, Griffith University, Parkland, Gold Coast, QLD 4222, Australia \\ ${ }^{2}$ Menzies Health Institute Queensland, Griffith University, Parkland, Gold Coast, QLD 4222, Australia \\ (Submitted 19 May 2015 - Final revision received 15 December 2015 - Accepted 23 December 2015)
}

\section{Abstract}

This meta-analysis examined the effect of probiotics on glucose and glycaemic factors in diabetes and its associated risk factors. All randomised-controlled trials published in English in multiple databases from January 2000 to June 2015 were systematically searched. Only studies that addressed glucose- and glycaemic-related factors as outcome variables were included. The main outcomes of interest in trials were mean changes in glucose, HbA1c, insulin and homoeostasis model assessment-estimated insulin resistance (HOMA-IR). Using the Physiotherapy Evidence Database (PEDro) scale to assess the quality of studies, a total of eleven studies with 614 subjects were included. The pooled mean difference and effect size with a $95 \%$ CI were extracted using a random-effect model. It was found that there are statistically significant pooled mean differences between the probiotics and the placebo-controlled groups on the reduction of glucose ( $-0.52 \mathrm{mmol} / \mathrm{l}$, $95 \%$ CI $-0.92,-0.11 \mathrm{mmol} / 1 ; P=0.01)$ and HbA1c $(-0.32 \%, 95 \%$ CI $-0.57,-0.07 \% ; P=0.01)$. There was no statistically significant pooled mean difference between the probiotics and the placebo-controlled groups on the reduction of insulin $(-0 \cdot 48 \mu \mathrm{IU} / \mathrm{ml}, 95 \% \mathrm{CI}-1 \cdot 34$, $0.38 \mu \mathrm{IU} / \mathrm{ml} ; P=0.27$ ) and HOMA-IR (pooled effect of $-0.44,95 \% \mathrm{CI}-1.57,0.70 ; P=0.45$ ). Meta-regression analysis identified that probiotics had significant effects on reduction of glucose, HbA1c, insulin and HOMA-IR in participants with diabetes, but not in participants with other risk factors. The present meta-analysis suggested that probiotics may be used as an important dietary supplement in reducing the glucose metabolic factors associated with diabetes.

\section{Key words: Probiotics: Glucose: Glycaemic factors}

The dramatic growth in the incidence of diabetes in recent years has led to increased efforts to find natural and safe strategies to control complications associated with the condition ${ }^{(1)}$. An abnormal metabolic profile, including impaired fasting glucose, insulin and glycaemic control, is a strong predictor of diabetes. Recently, it has been found that patients ${ }^{(1-3)}$ with type 2 diabetes show an alteration in their gut microbial composition $^{(4)}$. This suggests that probiotics may provide a new and promising way of regulating glucose and glycaemic factors through modifying gut microflora.

Probiotics have been defined by the WHO as live microorganisms which when administered in adequate amounts confer a health benefit on the host ${ }^{(5)}$. It has been demonstrated that probiotics can regulate gut microflora, which has health benefits in terms of improving gut health ${ }^{(6,7)}$ and regulating plasma lipids ${ }^{(8)}$. Furthermore, probiotics may have a role in preventing CVD and other chronic diseases through increasing enzymatic antioxidant activity, decreasing lipid components, BMI and blood pressure ${ }^{(9,10)}$. The multiple effects of probiotics raise questions as to whether they could aid the treatment of people with diabetes and associated risk factors.
Previous studies have assessed the effect of probiotics on metabolic profiles in people with hyperlipidaemia ${ }^{(11)}$ and healthy adults ${ }^{(12,13)}$, and a systematic review showed a moderate effect on glycaemic control in trials with a mixture of healthy and at-risk populations ${ }^{(14)}$. However, the impact of probiotics on the glycaemic control of diabetes or associated risk factors is uncertain. For example, there is conflicting evidence about the effects of probiotics on lowering glucose and glycaemic components ${ }^{(13)}$, with some studies finding them effective $^{(1-3)}$ while others not ${ }^{(1,12,15)}$. It is possible that multiple factors may confound the beneficial effects of probiotics, such as diabetic condition, single $v$. multiple species of probiotics, dosage use of probiotics, duration of probiotic consumption and probiotics in capsule or milk form. These factors need to be systematically examined to fully determine the effects of probiotics on lowering glucose and glycaemic factors in diabetes and its risk factors. The objective of the present meta-analysis was to synthesise results from randomised placebo-controlled studies on the effects of probiotics consumption in lowering glucose and glycaemic components in trials including both diabetes and risk factors of diabetes. These components

\footnotetext{
Abbreviation: HOMA-IR, homoeostasis model assessment-estimated insulin resistance.
}

* Corresponding author: Associate Professor J. Sun, fax +61 75678 0303, email j.sun@griffith.edu.au 
included glucose, HbA1c, insulin and homoeostasis model assessment-estimated insulin resistance (HOMA-IR).

\section{Methods \\ Literature search}

The review protocol was registered at Prospero International Prospective Register of Systematic Reviews (Registration ID = CRD42014013606 PROSPERO 2014 website: http://www.crd.york. ac.uk/PROSPERO/printPDF.php?RecordID=13606\&UserID=7309). The research team collected all pertinent studies published in English from 2000 to June 2015 through a systematic search of the following databases: PubMed, Scopus and the Cochrane Library. In addition, citation lists of all relevant articles and previous reviews were searched. Where a study had unreported data, the authors were contacted to determine whether the information was available. A combination of the following key words was used to locate relevant studies: Probiotics AND glycaemic factor or glucose OR insulin OR homeostasis model assessment and diabetes or diabetic risk factors. The title and abstract were first screened for relevance. The full text of the relevant articles was retrieved for further reading and quality assessment. Two researchers extracted data independently using a standard form and then resolved differences by discussion. The literature search and presentation of results were undertaken in accordance with the Preferred Reporting Items for Systematic Reviews and Meta-Analysis (PRISMA) guidelines. Identified articles were subsequently imported into EndNote reference package.

\section{Inclusion criteria}

The research team developed consensus on the four criteria used to determine the inclusion and exclusion of studies, and were as follows:

(1) Criterion 1: randomised placebo-controlled trials involved participants with diabetes or associated risk factors, including diabetes, abnormal glucose or HbA1c or insulin levels, overweight, obesity or the metabolic syndromes;

(2) Criterion 2: all trials involved human participants;

(3) Criterion 3: participants in the sample were randomly allocated into intervention (probiotics consumption) and control (placebo) groups, and all publications were quantitative research method based;

(4) Criteria 4: one or more of the following factors were included as outcome variables: glucose, HbA1c, insulin and HOMA-IR.

Where there were multiple publications and companion papers from the same population, only those papers with the largest sample and longest duration of intervention were included. In the event when there were multiple groups in one study, only the probiotics group and placebo group were included.

\section{Data extraction and quality assessment}

Extraction of data and assessment of studies according to the above-mentioned criteria were independently conducted by two researchers. The PRISMA flow chart was used to present a summary of the review (see Fig. 1). A pre-piloted data form was used to extract data from the selected randomised-controlled studies. Outcome variables extracted included glucose, HbA1c, HOMA-IR and insulin. Data were retrieved about the effects of probiotics on diabetes and groups with risk factors of diabetes and the characteristics of probiotics intake, including duration of probiotics intake, dosage of probiotics use, probiotics in milk or in capsule forms, single-strain probiotics intake and multiple strains of probiotics. Groups with risk factors of diabetes were defined as participants who had hypertension, hyperlipidaemia, obesity or other types of clinical illnesses. Data on glucose were converted to $\mathrm{mmol} / \mathrm{l}$ when they were reported as $\mathrm{mg} / \mathrm{dl}$ using the online converter: http://www.endmemo.com/medical/unitconvert/Glucose.php. The assessment of the abstracted articles was discussed with a view to gaining consensus. Disagreements were resolved through discussion.

The Physiotherapy Evidence Database (PEDro) tool was chosen to assess the quality of the abstracted articles (see Table 1$)^{(16)}$. This tool categorises the quality of the evidence into three levels (high, 8 or more points; moderate, 4-7 points; low, 3 points or less) based on ten factors including the following: (1) random allocation of subjects into groups, (2) concealed randomisation, (3) similarity of baseline information between groups, (4) blinding in relation to subjects, assessors, (5) blinding in relation to researchers, (6) blinding in relation to assessors, (7) low attrition rate, (8) use of 'intention to treat' analysis, (9) use of variability measures such as standard deviation and/or standard error and (10) betweengroup comparison results. One point is allocated for each item present in the study. Studies assessed as low quality were excluded from the review ${ }^{(16)}$.

\section{Statistical analysis}

The comprehensive meta-analysis for pooled mean difference and effect size was used to analyse the mean differences ${ }^{(17)}$. The effect measures pooled mean difference with a $95 \% \mathrm{CI}$ was calculated to describe the effects of probiotics on the outcome variables. Subgroup analyses were used to determine possible sources of heterogeneity. Studies with values $<50 \%$ were considered to have low heterogeneity. Randomeffect models were used to take into account between-study variation.

The detailed effect of probiotics was further explored using subgroup analysis to identify the sources of heterogeneity in relation to effects of (1) group with diabetes $v$. participants with risk factors of diabetes, (2) duration of probiotics intake (<8 weeks $v$. $\geq 8)$, (3) dosage level $\left(10^{9}\right.$ colony-forming units (CFU) or more $v$. less than $10^{9} \mathrm{CFU}$ use and fermented dairy products), (4) medium type (milk-based $v$. capsulebased); (5) single strain $v$. multiple strain and (6) age of the participants $(<50 v . \geq 50$ years). A multivariate meta-regression method was used to analyse the effects of probiotics due to multiple factors. Publication bias was assessed using funnel plots and Egger's regression test. A $P$ value of more than 0.05 in the Egger's regression test suggests that there is no 


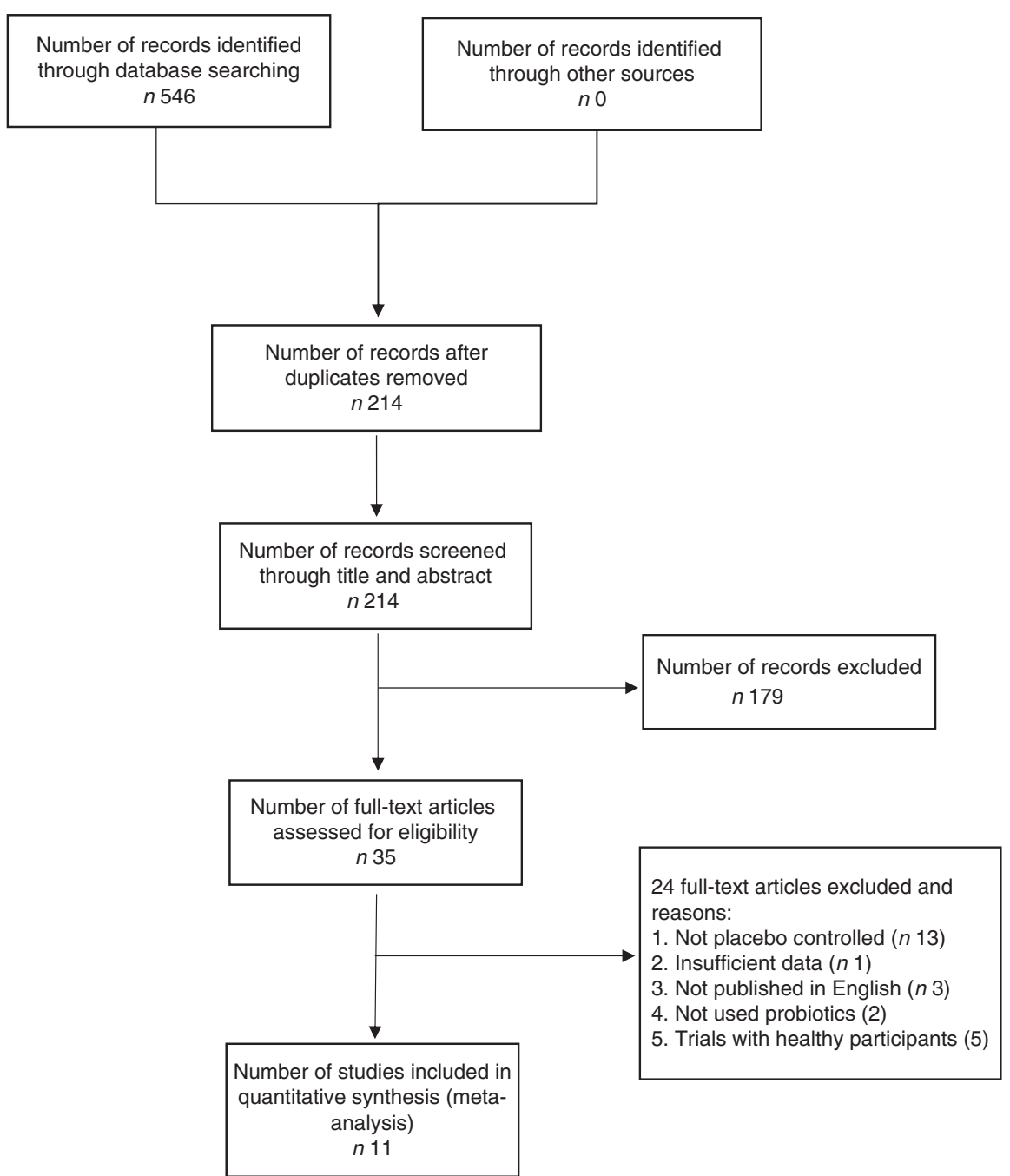

Fig. 1. Preferred Reporting Items for Systematic Reviews and Meta-Analysis flow chart of the included studies.

publication bias $^{(18)}$. Sensitivity analyses were conducted to assess whether the inferences overly depended on a particular study.

\section{Results}

In the initial search, 547 articles were identified from the key databases (220 from PubMed, 326 from Scopus and one from Cochrane Library) (see Fig. 1) and imported into EndNote. After removing duplicate studies, the remaining 211 were screened for relevance through their title and abstract, and then assessed according to the eligibility criteria previously stated. This step resulted in thirty-five articles. Further review using the PEDro tool resulted in twenty-three papers being excluded (ten because of a lack of placebo groups, six because of not focusing on diabetes or associated risk factors, two because of the language being other than English, four because of to low quality and two because of use of non-probiotic products). The remaining eleven papers were included in the final quantitative analysis, with ten having high quality ( 8 points or more) and one with moderate quality (5-7 points). Table 1 shows the final list of included studies, with their summary characteristics and quality assessment results.

\section{Description of the included studies}

A total of eleven randomised-controlled studies examining the effects of probiotics on the glucose and glycaemic factors among healthy participants and participants with diabetes or diabetic risk factors were included in the analysis (see Table 1), representing a total sample of 614 subjects. Among these studies, nine were double blinded ${ }^{(1,11,15,19-24)}$ and two were single blinded ${ }^{(3,25)}$. All studies were blinded to assessors. Samples were drawn from seven countries including Australia, Brazil, Denmark, Ireland, Iran, India and Russia. Participant characteristics included diabetes ${ }^{(1,3,11,21,22)}$, obesity and overweight $^{(15,19,20)}$, hypertension ${ }^{(24)}$ and the metabolic syndrome $^{(25)}$. All studies measured one or more of the following outcome variables: glucose, HbA1c, HOMA-IR and insulin. The summary description of the study characteristics is presented in Table 1. 


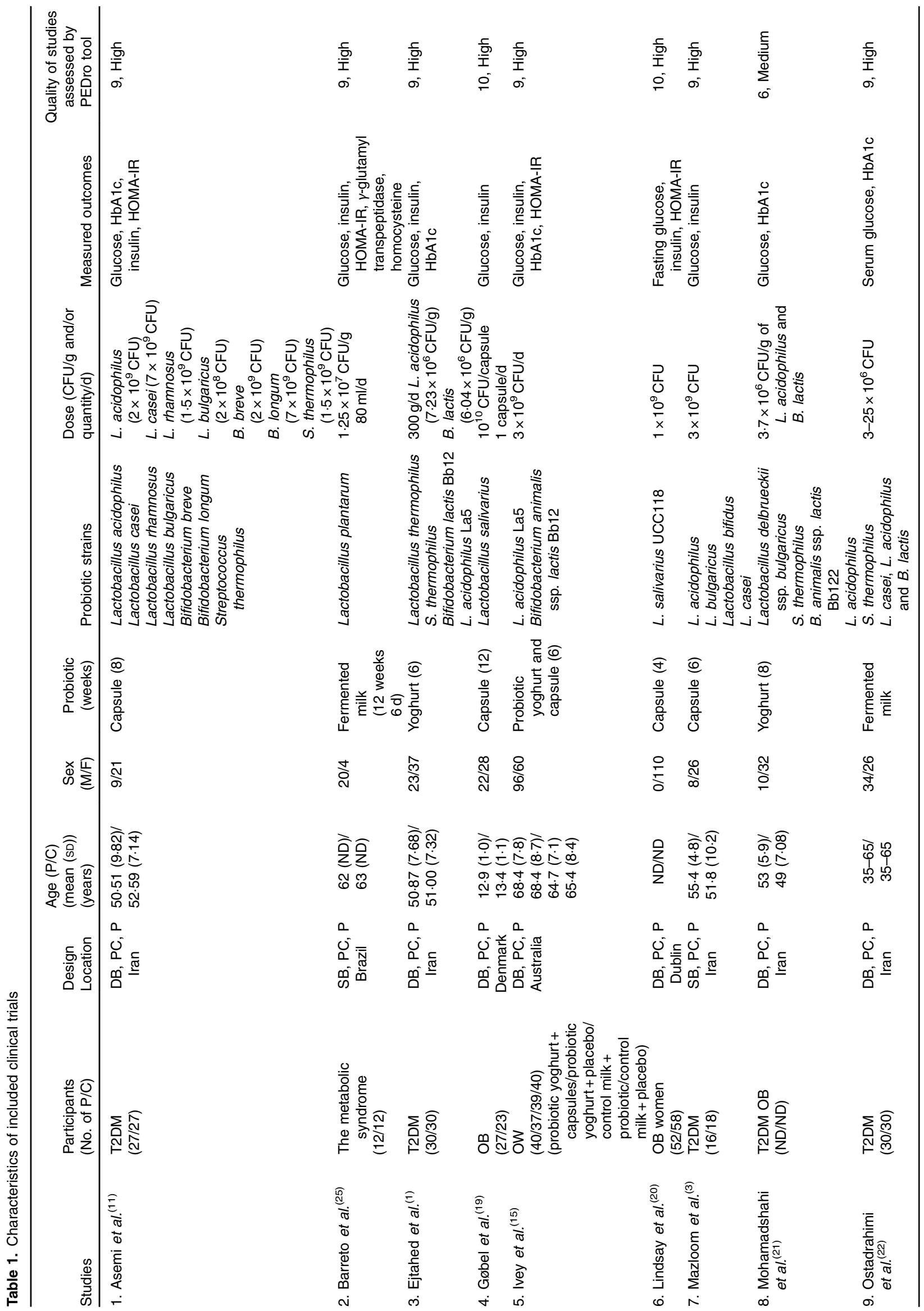




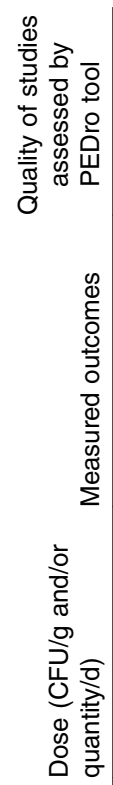

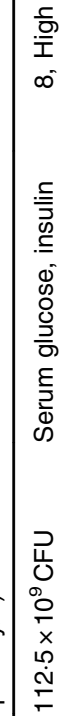
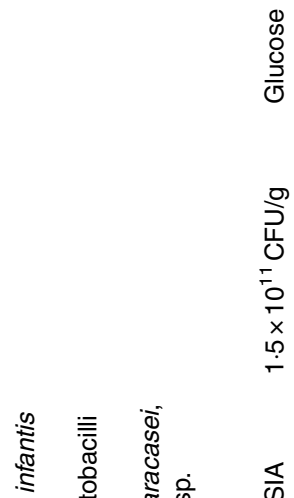

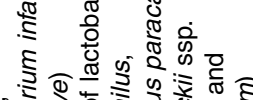

ब
0

0

ळ్m

$\frac{\widehat{N}}{\stackrel{N}{M}}$

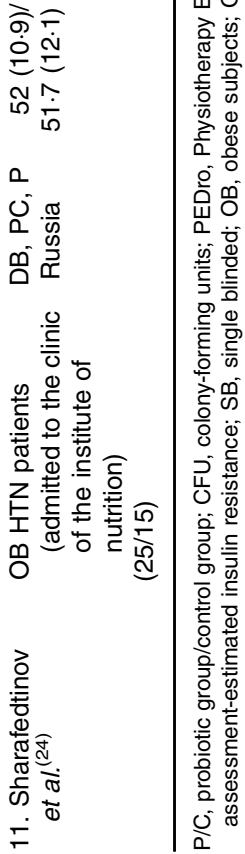

\section{Effects on fasting g/ucose}

All studies reported the effects of probiotics on fasting glucose (see Fig. 2(a)). The average reduction of glucose was $-0.50 \mathrm{mmol} / \mathrm{l}$ (range of 0.09 to $-1.29 \mathrm{mmol} / \mathrm{l}$ ) in the probiotics group compared with $0.13 \mathrm{mmol} / \mathrm{l}$ (range of 1.90 to $-1.20 \mathrm{mmol} / \mathrm{l})$ in the control group. The pooled effect was $-0.52 \mathrm{mmol} / \mathrm{l}(95 \% \mathrm{CI}-0.92,-0.11 \mathrm{mmol} / \mathrm{l} ; P=0.01)$ with a heterogeneity level $I^{2}=94 \%, P<0.001$.

Subgroup analyses revealed significant subgroup effects on glucose (Table 2). In these analyses, a probiotic diet resulted in decreased glucose only in trials on diabetes, with a pooled mean difference of $-1.46 \mathrm{mmol} / 1$ (95\% CI $-1.67,-1.26 \mathrm{mmol} / \mathrm{l}$; $P<0.001)$. The heterogeneity level was $I^{2}=0 \%, P=0.66$. There was no significant pooled mean difference and heterogeneity in trials among participants with other health conditions, with the pooled mean difference of $-0.20 \mathrm{mmol} / 1 \quad(95 \%$ CI -0.58 , $0.19 \mathrm{mmol} / \mathrm{l}, Z=-1.59 ; P=0.11), I^{2}=92 \%, P<0.001$. Multivariate meta-regression was further conducted to confirm the effects of probiotics on glucose in participants with diabetes when multiple factors including dosage of probiotics consumption, form of probiotics and number of strains were analysed simultaneously (Table 4). There was a statistically significant effect of probiotics on glucose and diabetes with an estimated coefficient of -1.28 (95\% CI $-3.82,-1 \cdot 25 ; P=0.03)$. In addition, the capsule form of probiotics consumption had more significant effects with an estimated coefficient of -2.61 (95\% CI $-4.74,-0.47 ; P=0.02)$. The total variances explained by the four variables were $100 \%$, of which diabetes status and form of probiotics explained a large proportion of the variances (43 and 48\%, respectively) (Fig. 2(a)).

\section{Effects on HbA1C}

Six studies reported HbA1c values among 348 participants (see Fig. 2(b)). The average reduction of HbA1c was 0.48 (range -0.3 to -1.21$) \%$ in the probiotics group compared with the increase of 0.05 (range 0.30 to -0.24 ) \% in the placebo group. There was a statistically significant reduction in $\mathrm{HbA1c}$ with a pooled mean difference of $-0.32 \%$ (95\% CI $-0.57,-0.07 \%$, $Z=2.49 ; P=0.01)$ and significant heterogeneity $\left(I^{2}=83 \%\right.$, $P<0.001$ ). Subgroup analysis (Table 2 ) was conducted, and it revealed that probiotics had a significant effect on the reduction of HbA1c in four diabetic trials among 218 participants ${ }^{(3,11,21,22)}$, with a pooled mean difference of $-0.52 \%(95 \%$ CI -0.71 , $-0.33 \% ; P<0.001)$ and heterogeneity $\left(I^{2}=5 \%, P=0.37\right)$. There was no significant effect of probiotics on the reduction of HbA1c in trials with other health conditions and no measurable heterogeneity $\left(I^{2}=0 \%, P=0.34\right)$ (Table 2$)$.

\section{Effects on insulin}

Eight studies, with 412 participants, reported the effects of probiotics on fasting insulin (see Fig. 2(c)). Probiotics had no statistically significant effect on fasting insulin levels in terms of a reduction in the probiotics group with a pooled mean difference of $-0.48 \mu \mathrm{IU} / \mathrm{ml}(95 \% \mathrm{CI}-1 \cdot 34,0.38 \mu \mathrm{IU} / \mathrm{ml} ; P=0.27)$ and significant heterogeneity $\left(I^{2}=92 \%, P<0.001\right)$. To assess 
(a)

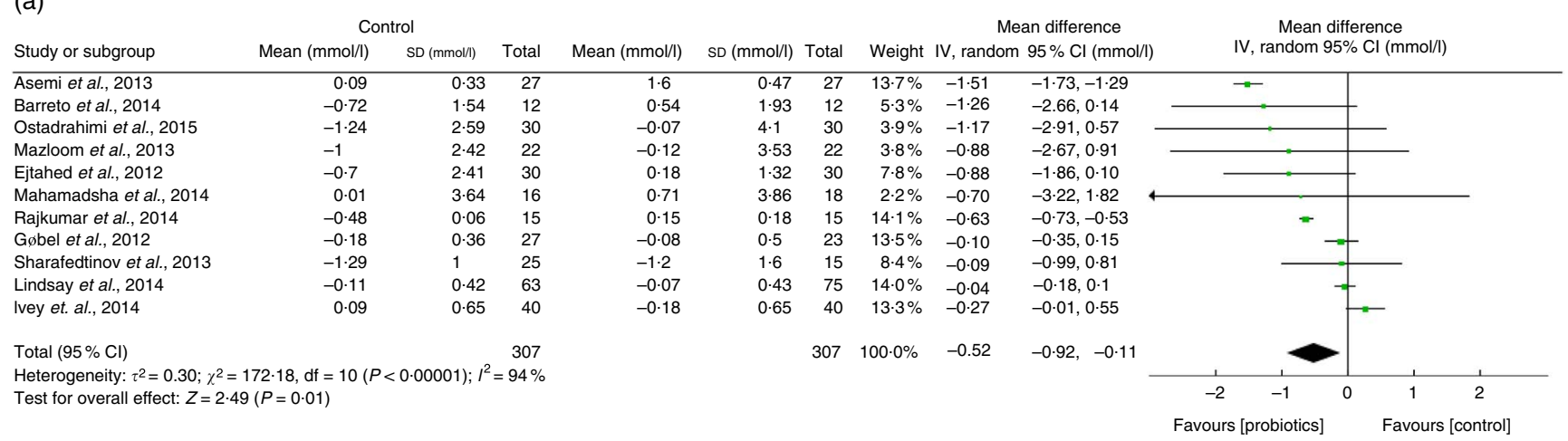

(b)

\begin{tabular}{|c|c|c|c|c|c|c|c|c|c|c|c|}
\hline \multirow[b]{2}{*}{ Study or subgroup } & \multicolumn{3}{|c|}{ Probiotics } & \multicolumn{3}{|c|}{ Control } & \multicolumn{3}{|c|}{ Mean difference } & \multirow{2}{*}{$\begin{array}{c}\text { Mean difference } \\
\text { IV, random } 95 \% \mathrm{Cl}(\%)\end{array}$} & \\
\hline & Mean (\%) & SD (\%) & Total & Mean (\%) & SD (\%) & Total & Weight & $\mathrm{V}$, rando & n $95 \% \mathrm{Cl}(\%)$ & & \\
\hline Ostadrahimi et al., 2015 & $-1 \cdot 21$ & 1.91 & 30 & 0.02 & 1.98 & 30 & $5 \cdot 3 \%$ & $-1 \cdot 23$ & $-2 \cdot 21,-0.25 \leftarrow$ & & \\
\hline Mahamadsha et al., 2014 & $-1 \cdot 15$ & $1 \cdot 23$ & 22 & -0.24 & 1.58 & 22 & $6 \cdot 8 \%$ & -0.91 & $-1.75,-0.07$ & & \\
\hline Asemi et al., 2013 & -0.3 & 0.37 & 27 & $0 \cdot 18$ & 0.31 & 27 & $24 \cdot 0 \%$ & $-0 \cdot 48$ & $-0.66,-0.30$ & $\rightarrow-$ & \\
\hline Ejtahed et al., 2012 & $-0 \cdot 12$ & $1 \cdot 24$ & 30 & 0.3 & 0.66 & 30 & $13 \cdot 1 \%$ & -0.42 & $-0.92,0.08$ & & \\
\hline Ivey et al., 2014 & -0.05 & 0.33 & 40 & 0.04 & 0.34 & 40 & $25 \cdot 1 \%$ & -0.09 & $-0.24,0.06$ & & \\
\hline Gøbel et al., 2012 & -0.03 & $0 \cdot 19$ & 27 & -0.03 & 0.22 & 23 & $25 \cdot 9 \%$ & 0.00 & $-0 \cdot 11,0 \cdot 11$ & & \\
\hline Total $(95 \% \mathrm{Cl})$ & & & 176 & & & 172 & $100 \cdot 0 \%$ & -0.32 & $-0.57,-0.07$ & & \\
\hline $\begin{array}{l}\text { Heterogeneity: } \tau^{2}=0.06 ; \chi \\
\text { Test for overall effect: } Z=\end{array}$ & $\begin{array}{l}=28.68, \mathrm{df} \\
49(P=0.0\end{array}$ & $5(P<0$ & $0.0001)$ & $2=83 \%$ & & & & & -2 & -1 & 2 \\
\hline
\end{tabular}

(c)

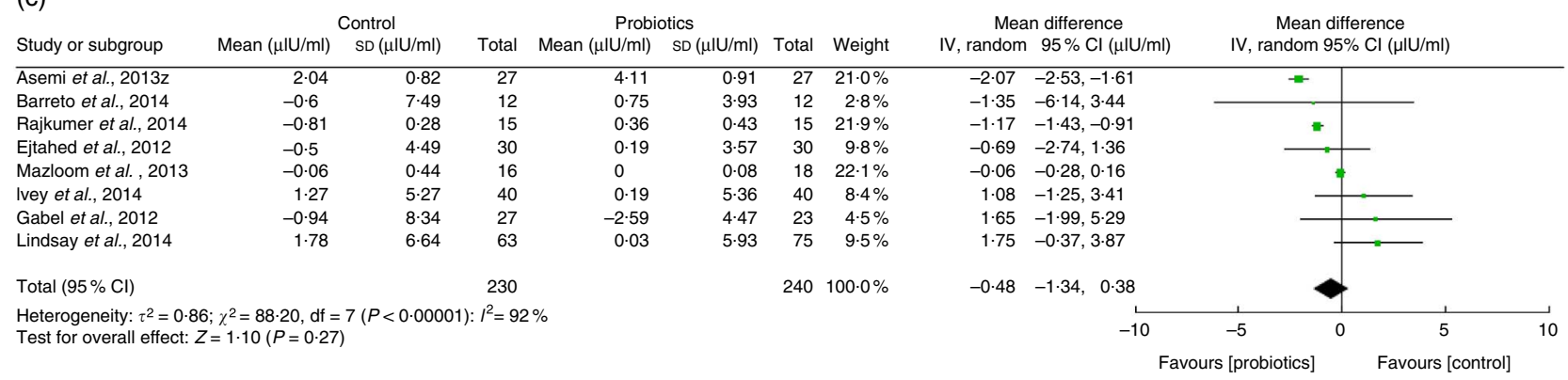

(d)

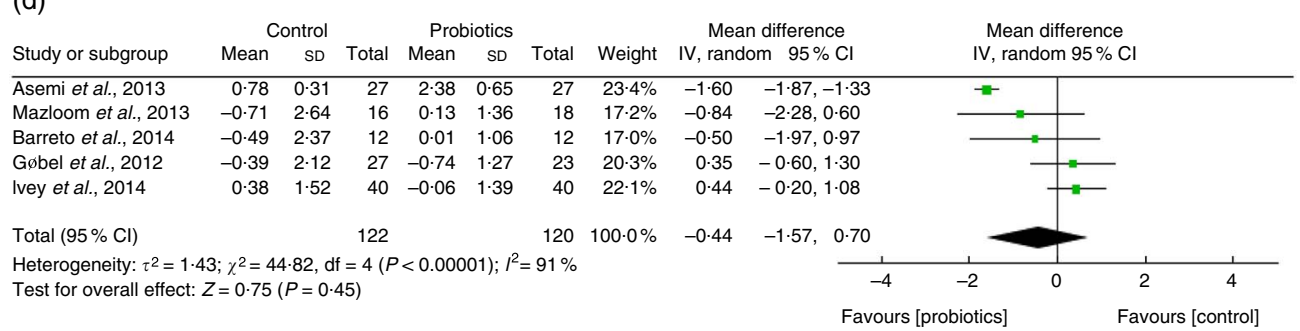

Fig. 2. Forest plots on (a) glucose, (b) HbA1c, (c) insulin and (d) homoeostasis model assessment-estimated insulin resistance.

the source of heterogeneity, subgroup analysis (Table 3) identified that probiotics had a significant effect on the reduction of insulin in the three diabetes trials involving 148 participants $^{(1,3,11)}$, with a pooled mean difference of $-0.40 \mu \mathrm{IU} / \mathrm{ml}$ (95\% CI $-0.59,-0.21 \mu \mathrm{IU} / \mathrm{ml} ; P<0.001)$ and significant heterogeneity $\left(I^{2}=96 \%, \quad P<0.001\right)$. There was also significant reduction in the trials among participants with other health conditions with a pooled mean difference of $-1 \cdot 11 \mu \mathrm{IU} / \mathrm{ml}(95 \%$ $\mathrm{CI}-1 \cdot 37,-0.85 \mu \mathrm{IU} / \mathrm{ml})$ and significant heterogeneity $\left(I^{2}=56 \%\right.$, $P<0 \cdot 001$ ) (Table 3).

\section{Effects on homoeostasis model assessment-estimated insulin resistance}

Eight studies reported HOMA-IR results among 242 subjects (see Fig. 2(d)). Probiotics had no statistically significant effect on the reduction in HOMA-IR (pooled effect of $-0 \cdot 44,95 \% \mathrm{CI}$ $-1 \cdot 57,0.70 ; P=0.45$ ). Subgroup analysis (Table 3 ) revealed that probiotics had a significant effect on the reduction of HOMA-IR in three diabetic trials among 138 participants with a pooled mean difference of -1.54 (95\% CI $-1.95,-1.13 ; P<0.001)$ and 
Probiotics 
there was no significant heterogeneity $\left(I^{2}=9 \%, \quad P=0 \cdot 29\right)$. There was no significant effect of probiotics on the reduction of HOMA-IR in other trials with a pooled mean difference of 0.31 (95\% CI $-0.20,0.81 ; P=0.23)$ with no measurable heterogeneity $\left(I^{2}=0 \%, P=0.51\right)$ (Table 3$)$.

\section{Subgroup, sensitivity and publication bias}

Subgroup analysis found that the effects of probiotic diet on glucose were statistically significant when it was in capsule form $(P<0.05)$ and with multiple strains. Consumption of probiotics with milk and single-strain consumption did not result in a significant reduction in glucose. Consumption of probiotics resulted in a significant reduction in glucose regardless of dosage level and consumption duration (Table 2).

Subgroup analysis found that the effects of probiotics diet on HbA1c and insulin were statistically significant when it was based on multiple strains of probiotics. Consumption of probiotics with a single strain did not result in a significant reduction in HbA1c or insulin levels (Tables 2 and 3). Furthermore, the effects of probiotic diet on insulin were statistically significant when it was in capsule form. Consumption of probiotics in milk form did not result in a significant reduction in insulin (Table 3).

Sensitivity analyses revealed that no particular study significantly affected the summary effects for glucose, HbA1c, insulin and HOMA-IR. The results for glucose, HbA1c, insulin and HOMA-IR showed minimal asymmetry, which indicates minimal publication bias. Visual inspection of funnel plots (Fig. 3) showed no clear evidence of publication bias with regard to effects on glucose, HbA1c, insulin and HOMA-IR. Findings from the Egger's regression test supported a finding that there was no publication bias for glucose $(P=0 \cdot 85)$, HbA1c $(P=0 \cdot 10)$, insulin $(P=0.57)$ and HOMA-IR $(P=0 \cdot 28)$ (Table 5$)$.

(a)

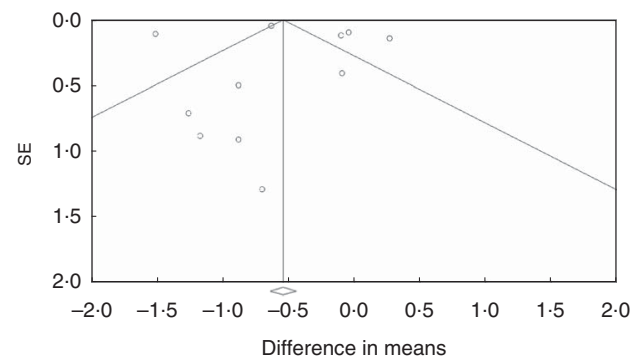

(c)

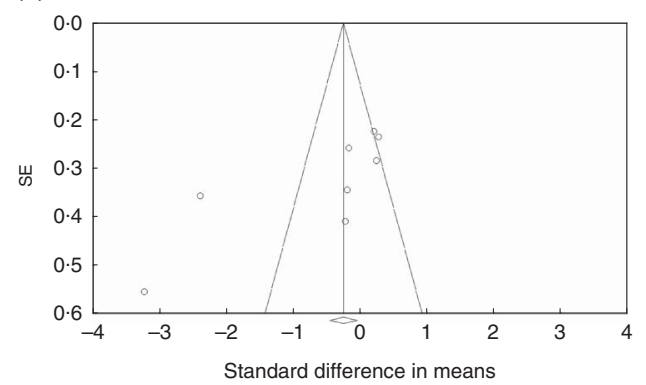

\section{Discussion}

Findings from this review indicate that probiotics consumption resulted in an overall reduction in glucose and HbA1c, but not in insulin and HOMA-IR, in trials with both diabetic participants and those with risk factors of diabetes. There were statistically significant reductions in glucose, HbA1c, insulin and HOMA-IR among participants with diabetes, with a large effect size, but not in glucose, HbA1c, HOMA-IR among those participants with risk factors of diabetes. This indicates that probiotics consumption has larger significant effects on reducing glucose metabolism in the diabetes population compared with populations who did not have elevated glycaemic levels. A similar result has also been observed in animal studies ${ }^{(26-28)}$.

One of the possible mechanisms by which this effect occurs is through the impact of probiotics on changing intestinal microbiota ${ }^{(4,29)}$. Following probiotics consumption, people with type 2 diabetes had balanced intestinal microbiota. This effect might have been caused by the SCFA that are produced from probiotics consumption $^{(30)}$ or decreased blood glucose due to increased gliclazide bioavailability ${ }^{(26)}$.

Probiotics are effective in suppression of the progression of streptozotocin-induced diabetes ${ }^{(28)}$. Streptozotocin has the ability to selectively kill pancreatic $\beta$ cells, which can result in a decrease in endogenous insulin release and increase of glucose intolerance ${ }^{(31)}$. Probiotics consumption containing Lactobacillus acidophilus and Lactobacillus casei can delay the progression of streptozotocin-induced diabetes by suppressing the increase in glucose intolerance and blood glucose and maintaining the insulin levels. This indicates that probiotic consumption may have an anti-diabetic effect through a role in protecting pancreatic b-cells from damage ${ }^{(28)}$.

Improvements in antioxidant stress level through probiotics consumption may also indirectly affect insulin level and glucose

(b)

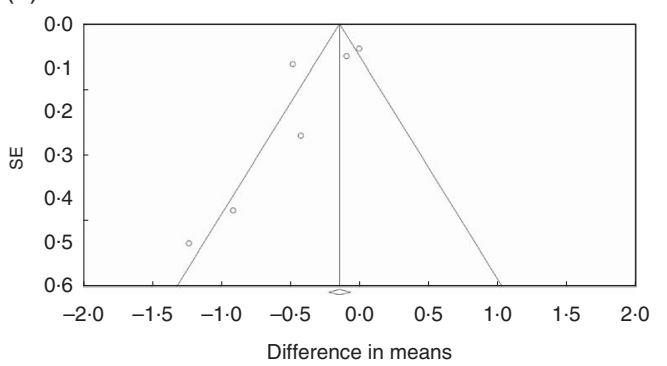

(d)

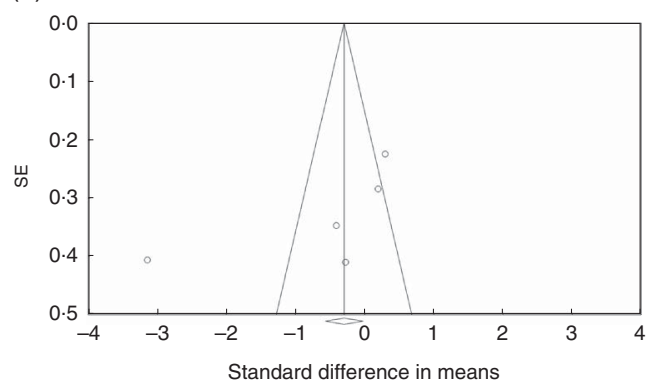

Fig. 3. Funnel plots on (a) glucose, (b) HbA1c, (c) insulin and (d) homoeostasis model assessment-estimated insulin resistance. 
Table 4. Meta-regression analysis (Mean differences and 95\% confidence intervals)

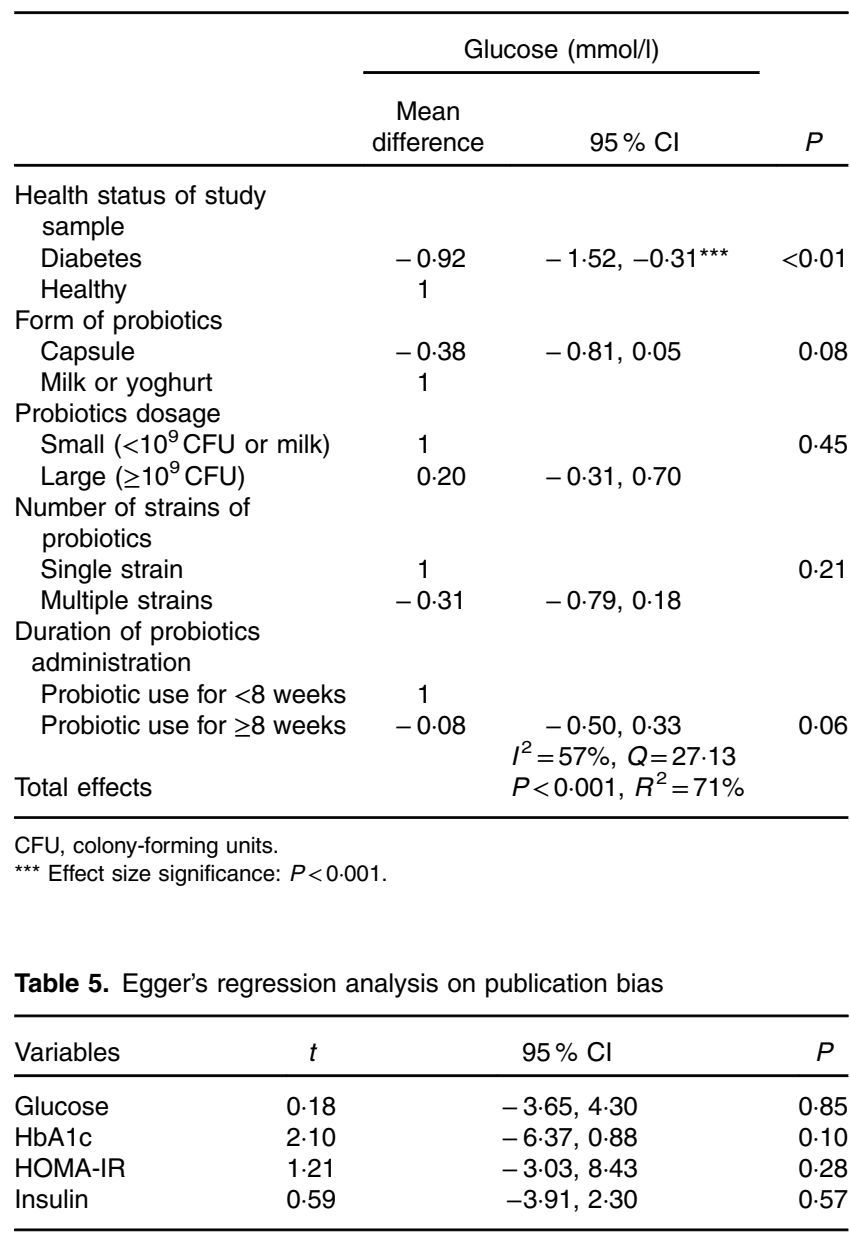

HOMA-IR, homoeostasis model assessment-estimated insulin resistance.

homoeostasis ${ }^{(1)}$. The distinct effect of probiotics consumption in relation to participants with diabetes may be because probiotics consumption can balance microflora in the gastrointestinal tract to normal levels, as well as change the intestine microbiota so that the elevation in insulin levels is delayed or prevented. In addition, it can also regulate abnormal glucose homoeostasis of participants with diabetes to the normal level. Although the exact mechanism in humans remains unclear, it is likely that multiple factors contribute to the effects of probiotics on diabetic patients ${ }^{(1)}$.

The number of strains used in the included studies was related to the effects of probiotics on the glucose and glycaemic factors. Seven trials for glucose, five trials for HbA1c (five out of six) and six trials (six out of eight) for insulin used multiple strains. It was found that trials with multiple strains had statistically significant reductions in HbA1c and insulin levels, whereas trials with single strains did not have significant reductions in HbA1c and insulin levels. The possible mechanism might be that these multiple strains regulate multiple systems through the production of SCFA in the gut, leading to reductions in exogenous cholesterol and LDL and glucose ${ }^{(32,33)}$. This result is similar to the finding that trials with multiple strains had better effects as compared with those with a single strain on blood pressure in a recent meta-analysis ${ }^{(9)}$. Owing to the limited number of trials with multiple strains, further studies are warranted to confirm these findings.

The form of probiotics was also related to the effect of probiotics on the outcome variables. Five trials (five out of eleven) for glucose and five trials (five out of eight) for insulin used the capsule form of probiotics. It was found that trials using capsules had significant reductions in glucose and insulin, whereas trials using the milk form did not have significant reduction in glucose and insulin. The possible reason might be that the trials using capsules might have resulted in better digestion of the product, and therefore led to higher levels of bioactivity, which may account for this result. The dosage level and duration of probiotics consumption did not have any effects. Four trials (four out of six) for HbA1c used the milk or yoghurt form of probiotics $^{(1,15,21,22)}$. It was found that trials using milk or yoghurt had a significant reduction in HbA1c. It is possible that the milk or yoghurt form may keep more bacteria alive in the tract to balance the HbA1c. The replacement of sugar in milk or yoghurt by probiotics does not account for the reduction in HbA1c, as both the probiotics and placebo groups had the similar ingredients, except for the addition of probiotics in the probiotics group. This finding suggests that the reduction of HbA1c is not due to the reduction of sugar levels in milk or yoghurt, but is a treatment effect of the probiotics per se. We do not know the effect of insulin $v$. probiotics on the reduction of glucose, HbA1c, insulin and HOMA-IR, as the studies reviewed in this meta-analysis excluded patients using insulin ${ }^{(1,3,11,21,22)}$. However, the findings cannot be accounted for by the effect of other anti-diabetic medications rather than probiotics. Despite the fact that some patients were using anti-diabetic medication in a number of studies, the probiotic and placebo groups were randomised and showed similar baseline results on demographic factors, anthropometry, glycaemic characteristics and medication use, thus ruling out the hypothesis that the treatment effect was due to medication rather than probiotics.

None of the studies in this meta-analysis reported any adverse side-effects related to the mediums used (fermented milk and capsules), indicating that they were safe to use. In addition, all the studies had $<15 \%$ attrition rate over the course of the intervention, suggesting that the subjects had excellent level of compliance with the consumption of probiotics. Probiotics consumption may have provided additional health benefits in addition to medication use.

\section{Strengths and limitations}

This is the first meta-analysis study on randomised-controlled trials (RCT) relating to the effect of probiotics on glucose and glycaemic factors. The strength of this study is that all studies published since 2000 to 2015 have been included in the meta-analysis, and where there were unreported data the authors were contacted to provide it. Therefore, all available data were included $^{(9)}$. All included studies in this review were placebo-controlled trials; thus, the effect of intervention due to probiotics consumption could be examined. In addition, the results of these analyses may be viewed as accurate, as we were able to adjust for multiple variables using meta-regression on glucose when conducting subgroup analyses. 
A number of limitations of this study should be noted. The use of strains of probiotics was not homogeneous across studies and some studies used strains that could sensitively regulate glucose metabolism, whereas others were not in the included clinical trials. Owing to the limited number of included trials, it was not possible identify the effect of specific strains of probiotics on the reduction of glucose, HbA1c, insulin and HOMA-IR. Further exploration of such subgroup differences within the context of RCT will entail a meta-analysis to identify the effect of specific strains on the reduction of glucose, HbA1c, insulin and HOMA-IR. We also recognise that studies published in languages other than English were not included, and thus it is unknown whether results of these studies would have had effects on our meta-analysis results.

\section{Implications for treatment and future research}

The findings of this meta-analysis suggest that probiotics may play an important role in the future prevention of diabetes and reduction of risk factors of diabetes. However, further research is needed to confirm its benefits in the prevention and treatment of diabetes, particularly in relation to form, variability of probiotic strains and specification of strains. Nevertheless, there is a recognised and important role played by probiotics and considerable potential benefits in managing a number of risk factors of diabetes. Furthermore, inadequate data sources in studies for insulin and HOMA-IR meant that we could not differentiate between subgroup differences such as duration of probiotics use, dosage, single-strain $v$. multi-strain effects and specific strain effects. Furthermore, RCT involving long-term interventions with single $v$. multiple strains of probiotic supplement regimens are required to further clarify these findings.

Future research using a RCT with a large sample size is needed to confirm that such alternative nutrition regimens are effective with regard to the reduction of glycaemic factors. Similarly, it is necessary to identify and characterise known and unknown strains of probiotics, and identify strain-specific health outcomes to optimise treatment effects. This will enhance our understanding of the application of probiotics in diabetes prevention and treatment. It is possible that probiotics can be used as an adjunct method for glycaemic control in addition to medication intake for patients with diabetes. There is potential in the field of translational diabetic medicine to further examine the role probiotics play as an important dietary supplement for the prevention of diabetes and the risk factors. Further research is needed on whether probiotics have a treatment effect, in addition to pharmaceutical drug administration, and to determine whether probiotics should be included as a functional food and treatment option for diabetes.

\section{Conclusions}

In summary, the findings of the present study suggest that probiotic supplementation use is effective in improving glucose metabolism. The effect of probiotics use on glucose is more effective when it is used in the capsule form and with multiple strains, and it is more effective in participants with impaired glucose and insulin resistance levels. With the improvement of multiple risk factors of diabetes, probiotics may provide a potential avenue to improve glucose metabolism, which may lead to a reduction in diabetes complications and its rising incidence.

\section{Acknowledgements}

The authors acknowledge Michelle Carty's assistance for the data search and extraction procedure.

This research received no specific grant from any funding agency, commercial or not-for-profit sectors.

J. S. designed the study, formulated research questions, collected data, analysed the data and wrote the article. N. J. B. collected data, evaluated the quality of the study, interpreted the data and edited the article.

The authors declare that there are no conflicts of interest.

\section{References}

1. Ejtahed HS, Mohtadi-Nia J, Homayouni Rad A, et al. (2012) Probiotic yogurt improves antioxidant status in type 2 diabetic patients. Nutrition 28, 539-543.

2. Asemi Z, Samimi M, Tabassi Z, et al. (2013) Effect of daily consumption of probiotic yoghurt on insulin resistance in pregnant women: a randomized controlled trial. Eur J Clin Nutr 67, 71-74.

3. Mazloom Z, Yousefinejad A \& Dabbaghmanesh MH (2013) Effect of probiotics on lipid profile, glycemic control, insulin action, oxidative stress, and inflammatory markers in patients with type 2 diabetes: a clinical trial. Iran J Med Sci $\mathbf{3 8}$, $38-43$.

4. Chin J (2005) Prospects for beneficial health outcomes from intestinal microflora. Asia Pac J Clin Nutr 14, 64-65.

5. Gilliland SE, Morelli L \& Reid G (2001) Health and Nutritional Properties of Probiotics in Food Including Powder Milk with Live Lactic Acid Bacteria. Report of a joint FAO/WHO expert consultation on evaluation of health and nutritional properties of probiotics in food including powder milk with live lactic acid bacteria. Rome, Italy: Food and Agriculture Organization of the United Nations World Health Organization. ftp://ftp.fao.org/es/ esn/food/probio_report_en.pdf (accessed February 2016).

6. Fooks LJ \& Gibson GR (2002) Probiotics as modulators of the gut flora. Br J Nutr 88, Suppl. 1, S39-S49.

7. Kaushik JK, Kumar A, Duary RK, et al. (2009) Functional and probiotic attributes of an indigenous isolate of Lactobacillus plantarum. PLOS ONE 4, e8099.

8. Guo C \& Zhang L (2010) Cholesterol-lowering effects of probiotics - a review. Wei Sheng Wu Xue Bao 50, 1590-1599.

9. Khalesi S, Sun J, Buys N, et al. (2014) Effect of probiotics on blood pressure: a systematic review and meta-analysis of randomized, controlled trials. Hypertension 64, 897-903.

10. Sun J \& Buys N (2015) Effects of probiotics consumption on lowering lipids and CVD risk factors: a systematic review and meta-analysis of randomized controlled trials. Ann Med $\mathbf{4 7}$, 430-440.

11. Asemi Z, Zare Z, Shakeri H, et al. (2013) Effect of multispecies probiotic supplements on metabolic profiles, hs-CRP, and oxidative stress in patients with type 2 diabetes. Ann Nutr Metab 63, 1-9.

12. Chang BJ, Park SU, Jang YS, et al. (2011) Effect of functional yogurt NY-YP901 in improving the trait of metabolic syndrome. Eur J Clin Nutr 65, 1250-1255.

13. Jones ML, Martoni CJ, Di Pietro E, et al. (2012) Evaluation of clinical safety and tolerance of a Lactobacillus reuteri NCIMB 30242 supplement capsule: a randomized control trial. Regul Toxicol Pharmacol 63, 313-320. 
14. Ruan Y, Sun J, He J, et al. (2015) Effect of probiotics on glycemic control: a systematic review and meta-analysis of randomized, controlled trials. PLOS ONE 10, e0132121.

15. Ivey KL, Hodgson JM, Kerr DA, et al. (2014) The effects of probiotic bacteria on glycaemic control in overweight men and women: a randomised controlled trial. Eur J Clin Nutr $\mathbf{6 8}$, 447-452.

16. Verhagen AP, de Vet HC, de Bie RA, et al. (1998) The Delphi list: a criteria list for quality assessments of randomised clinical trials for conducting systematic reviews developed by Delphi consensus. J Clin Epidemiol 51, 1235-1241.

17. Biostat (2013) Comprehensive Meta-Analysis. Englewood, NJ: Biostat.

18. Egger M, Smith GD, Schneider M, et al. (1997) Bias in metaanalysis detected by a simple, graphical test. BMJ 315, 629-634.

19. Gøbel RJ, Larsen N, Jakobsen M, et al. (2012) Probiotics to adolescents with obesity: effects on inflammation and metabolic syndrome. J Pediatr Gastroenterol Nutr 55, 673-678.

20. Lindsay KL, Kennelly M, Culliton M, et al. (2014) Probiotics in obese pregnancy do not reduce maternal fasting glucose: a double-blind, placebo-controlled, randomized trial. Am J Clin Nutr 99, 1432-1439.

21. Mohamadshahi M, Veissi M, Haidari F, et al. (2014) Effects of probiotic yogurt consumption on lipid profile in type 2 diabetic patients: a randomized controlled clinical trial. J Res Med Sci 19, 531-536.

22. Ostadrahimi A, Taghizadeh A, Mobasseri M, et al. (2015) Effect of probiotic fermented milk (kefir) on glycemic control and lipid profile in type 2 diabetic patients: a randomized doubleblind placebo-controlled clinical trial. Iran J Public Health $\mathbf{4 4}$, 228-237.

23. Rajkumar H, Mahmood N, Kumar M, et al. (2014) Effect of probiotic (VSL\#3) and omega-3 on lipid profile, insulin sensitivity, inflammatory markers, and gut colonization in overweight adults: a randomized, controlled trial. Mediators Inflamm 2014, 348959 .
24. Sharafedtinov K, Plotnikova O, Alexeeva R, et al. (2013) Hypocaloric diet supplemented with probiotic cheese improves body mass index and blood pressure indices of obese hypertensive patients - a randomized double-blind placebo-controlled pilot study. Nutr J 12, 138.

25. Barreto FM, Colado Simão AN, Morimoto HK, et al. (2014) Beneficial effects of Lactobacillus plantarum on glycemia and homocysteine levels in postmenopausal women with metabolic syndrome. Nutrition 30, 939-942.

26. Al-Salami H, Butt G, Fawcett JP, et al. (2008) Probiotic treatment reduces blood glucose levels and increases systemic absorption of gliclazide in diabetic rats. Eur J Drug Metab Pharmacokinet 33, 101-106.

27. Harisa GI, Taha EI, Khalil AF, et al. (2009) Oral administration of Lactobacillus acidophilus restores nitric oxide level in diabetic rats. Aust J Basic Appl Sci 3, 2963-2969.

28. Yadav H, Jain S \& Sinha PR (2008) Oral administration of dahi containing probiotic Lactobacillus acidophilus and Lactobacillus casei delayed the progression of streptozotocin-induced diabetes in rats. J Dairy Res 75, 189-195.

29. Larsen N, Vogensen FK, van den Berg FWJ, et al. (2010) Gut microbiota in human adults with type 2 diabetes differs from non-diabetic adults. PLOS ONE 5, e9085.

30. Haleh Sadrzadeh-Yeganeh H, Ibrahim Elmadfa I, Abolghasem Djazayery A, et al. (2010) The effects of probiotic and conventional yoghurt on lipid profile in women. BrJ Nutr $\mathbf{1 0 3}$, $1778-1783$.

31. Wilson GL, Patton NJ, McCord JM, et al. (1984) Mechanisms of streptozotocin and alloxan induced damage in rat beta cells. Diabetologia 27, 587-596.

32. Pereira DIA \& Gibson GR (2002) Effects of consumption of probiotics and prebiotics on serum lipid levels in humans. Crit Rev Biochem Mol Biol 37, 259-281.

33. Wong JM, de Souza R, Kendall CW, et al. (2006) Colonic health: fermentation and short chain fatty acids. J Clin Gastroenterol 40, 235-243. 